\title{
Dielectric study of electrolyzed rubber
}

\begin{abstract}
Rubber was produced by placing rubber latex between two electrodes between which an external voltage was maintained. The thickness of the rubber deposited on the anode was measured as a function of time of electrolysis, voltage applied across the electrodes and distance between the electrodes. Based on the observed dielectric responses of rubber obtained through the electrolysis process, an electrical model of conductance and complex capacitance elements linked in parallel is proposed, indicating the additive nature of the individual responses in the system. Electrical representation of rubber obtained by simple drying however showed two different models. Natural rubber was found to have all the elements connected in parallel while that of vulcanized rubber was found to have a series connection of a pair of parallel connected conductance and capacitance elements.
\end{abstract}

Keyword: Rubber; Dielectric; Drying; Electrolyzed rubber 